\title{
Adenoma nefrogénico prostático. A propósito de un caso
}

\section{Prostatic nephrogenic adenoma. A case report}

\author{
R. Algarra, J. F. Barba, L. M. Romero, A. Tienza, A. Panizo, J. M. Berián
}

\section{Sr. Director:}

El adenoma nefrogénico es una lesión pseudotumoral rara de origen urotelial que afecta preferentemente a la vejiga urinaria, y que ha recibido varios nombres en la literatura. Se caracteriza por ser una entidad benigna asociada a ciertos estados irritativos de la vía urinaria. Debido a ello, se ha sugerido que su naturaleza puede corresponder a una metaplasia del urotelio.

Existen pocos casos documentados de adenoma nefrogénico de localización prostática (metaplasia nefrogénica).

Nuestro objetivo es poner en relevancia la rareza de esta patología y sus implicaciones terapéuticas.

Acude a consulta un paciente de 65 años sin antecedentes médicos de interés, diagnosticado de hiperplasia benigna de próstata con PSA $1,4 \mathrm{ng} / \mathrm{ml}$. en tratamiento con Tamsulosina y Finasteride. El tacto rectal revela una próstata grado III/IV, fibroelás- tica, no dolorosa y asimétrica a expensas del lóbulo izquierdo, con una volumen de unos $80 \mathrm{cc}$ hallados en la ecografía. Ante la persistencia de la sintomatología urinaria obstructiva se le indica fotovaporización prostática con láser KTP practicándose sin incidencias.

A los 7 meses acudió a consulta por episodio de hematuria macroscópica acompañado de disuria. Dado que el sedimento y cultivo resultaron anodinos, se le realizó una uretrocistocopia en la que se apreció la existencia de un esferoide residual prostático a nivel del cuello vesical. De acuerdo a los hallazgos y la clínica asociada se le propuso tratamiento quirúrgico realizándose una resección transuretral (RTU) de dicho esferoide residual.

El informe anatomopatológico informó de hiperplasia de próstata y adenoma nefrogénico (metaplasia nefrogénica). (Figs. 1-3)
Clínica Universidad de Navarra. Departamento de Urología.

Recepción: 17 de mayo de 2011

Aceptación provisional: 21 de junio de 2011

Aceptación definitiva: 22 de junio de 2011

\author{
Correspondencia: \\ Clínica Universidad de Navarra \\ Departamento de Urología \\ Avda. Pío XII, 36 \\ 31008 Pamplona \\ E-mail: ralgarra@unav.es
}




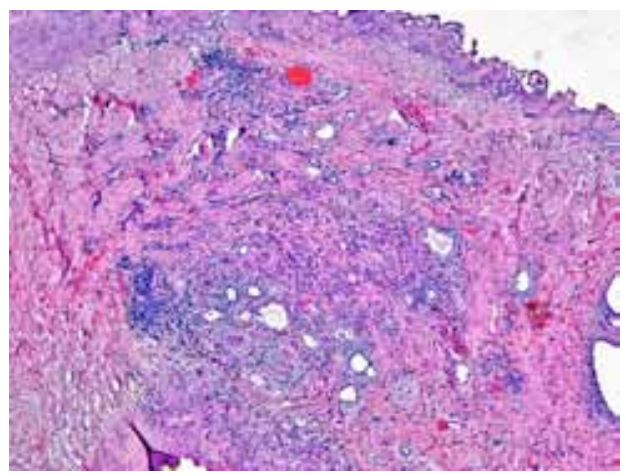

Figura 1. Lesión de naturaleza epitelial en parte papilar exofítica y en parte con un crecimiento entre el músculo liso del parénquima prostático (hematoxilina-eosina).

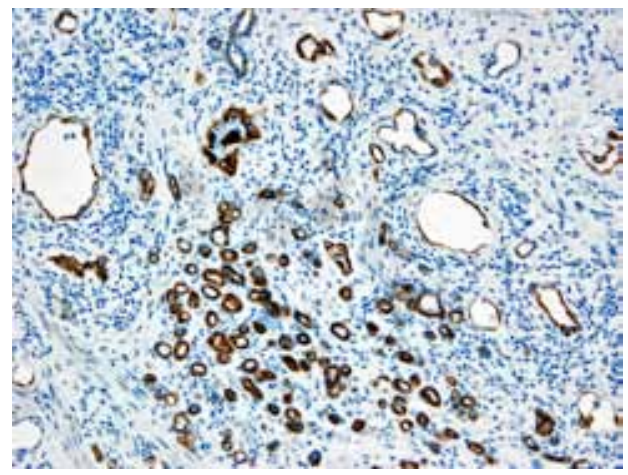

Figura 3. Células basales marcadas positivamente con el marcador de la citoqueratina CK $34 \mathrm{bE} 12$.

Tras la retirada de la sonda, el paciente fue dado de alta en buen estado general y logrando micciones espontaneas.

El adenoma nefrogénico fue descrito inicialmente por Davis en 1949 quien lo interpretó debido a su semejanza histológica con los túbulos renales como un hamarto$\mathrm{ma}^{1-3}$.

Friedman y Kuhlenbeck acuñaron dicho término para denominar a una lesión que presentaban sus pacientes a nivel vesical caracterizada por la presencia de una lesión adenomatoide que recordaba a la nefrona distal ${ }^{4,5}$.

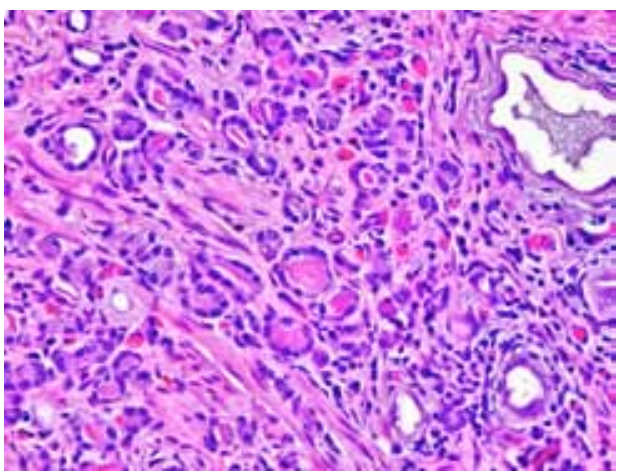

Figura 2. Presencia de material de secreción basófilo denso en la luz de las estructuras tubulares (hematoxilina-eosina).

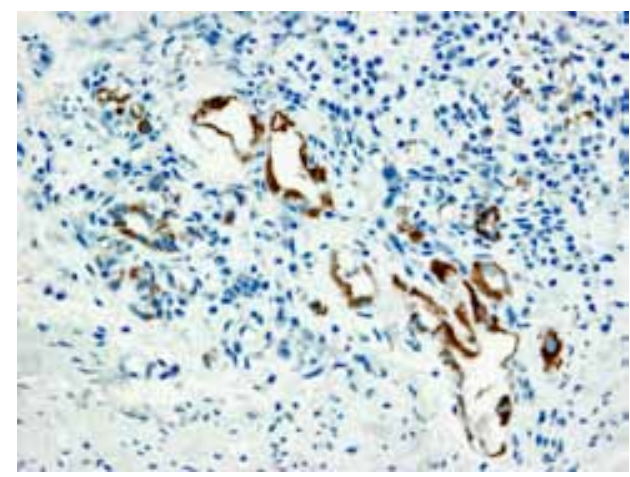

Figura 4: Débil inmunotinción para racemasa. No se observa atipia, mitosis, necrosis $u$ otros signos de malignidad.

Su ultraestructura recuerda más a la de un simple y primitivo túbulo mesonéfrico que a los complejos y evolucionados túbulos metanéfricos. Esta hipótesis parece confirmarse por el hecho de que el adenoma nefrogénico muestra una ausencia de la proteína de Tamm-Horsfall en la luz tubular o en las células que la tapizan, mientras que dicha proteína sí está presente en el segmento grueso ascendente del asa de henle y en el túbulo contorneado distal cuyos orígenes son metanéfricos ${ }^{4-8}$.

$\mathrm{Su}$ frecuencia es aproximadamente 3 o 4 veces mayor en varones, hecho que po- 
dría apoyar un posible origen a partir del conducto mesonéfrico o de Wolf, ya que en la mujer estos conductos degeneran. Sin embargo, en la infancia la proporción se invierte siendo casi 4 veces más frecuente en las niñas y está asociado además a malformaciones congénitas locales como el reflujo vesico-ureteral o divertículos uretrales $^{4,9-11}$.

Puede localizarse en cualquier parte del tracto urinario, siendo el trígono vesical el lugar más frecuente con un $80 \%$, seguido de uretra $15 \%$, uréter $5 \%$ y rara vez se encuentra en pelvis renal ${ }^{4,12}$.

Su origen es metaplásico, pudiendo remedar una neoplasia maligna tanto en el estudio endoscópico como en el histológico.

Más de la mitad de los casos descritos hasta la fecha están relacionados con cirugías previas $^{13}$, por lo que pueden ser etiquetadas erroneamente como recidivas neoplásicas en cistoscopias de seguimiento post-RTU. También se ha encontrado relación con traumatismos, estados de inmunosupresión como en pacientes trasplantados $^{14,15}$ y estados inflamatorios crónicos como infecciones urinarias recurrentes o secundario a instilaciones intravesicales con Bacillus de Calmette y Guérin $(\mathrm{BCG})^{16,17}$.

Puede mostrar varios aspectos morfológicos, algunos de ellos muy semejantes a una neoplasia maligna, o en forma de lesión plana similar a la del carcinoma in situ o invasor. También puede mostrar patrones similares al adenocarcinoma de células claras y el carcinoma de células transicionales con patrón de nidos sólidos. En el estudio endoscópico podemos encontrar lesiones de aspecto papilar en un $55 \%$, en un $10 \%$ son formaciones polipoideas, y hasta en un $35 \%$ aparecen con morfología sesil.

Histológicamente se caracteriza por formaciones tubulares y quistes cubiertos por una capa única de células planas, cúbicas, columnares o en tachuela con escaso citoplasma eosinofílico, amfofílico o claro $^{1}$.

En el caso del carcinoma, las células presentan atipia, mitosis, necrosis, y positividad para p53, MIB1 y otro tipo de marcadores $^{18}$.
En la mayoría de los casos, el diagnóstico de adenocarcinoma mínimo es posible a partir de la utilización de críterios histológicos mayores como son los siguientes: acinos que infiltran el estroma prostático particularmente entre las glándulas benignas, atipia nuclear asociada o no a macronucleolos prominentes, citoplasma basófilo, y ausencia de la capa de las células basales. Únicamente un número limitado de rasgos -tales como las glomerulaciones, la fibroplasia mucinosa (micronódulos de colágeno) y la invasión perineural- son patognomónicos de cáncer de próstata ${ }^{19-21}$.

No obstante a partir del mero examen de las preparaciones es difícil proporcionar un diagnóstico inequívoco de malignidad, y especialmente en el caso de focos limitados o lesiones acinares atípicas de pequeño tamaño. En dichas circunstacias, la tinción inmunohistoquímica puede resultar de utilidad.

Los marcadores de células basales, tales como la citoqueratina (CK) de alto peso molecular 903 (34bE12), la CK 5/6 y el p63, subrayan la presencia de células basales en las glándulas benignas. Esas células se hallan ausentes en los carcinomas de próstata $^{19,22-25}$. Sin embargo Yang y col detectaron positividad a $34 \mathrm{bE} 12$ en el $2 \%$ de los cánceres de próstata metastásicos donde la inmunorreactividad se encontró restringida a un tipo raro de células tumorales de alto grado ${ }^{26}$. En contrapartida, una tinción negativa para células basales en unas pocas células sospechosas no es una prueba definitiva de malignidad, dado que algunas entidades benignas pueden exhibir una distribución de células basales parcheada o discontinua ${ }^{27}$.

Por esas razones la identificación del marcador de cáncer de próstata a-metilacil- Co A racemasa (AMACR) constituye una aportación verdaderamente afortunada $^{28}$. Han sido identificados tres genes P503s, P504s, P501s responsables de la expresión de esta coenzima. Concretamente se han generado anticuerpos monoclonales de conejo específico demostrando inmurreactividad frente a P504s en el carcinoma de próstata. Dicha enzima interviene en el metabolismo lipídico tisular de 
manera que su aumento de expresión podría representar un cambio preneoplásico temprano y podría reflejar una regulación al alza de los patrones metabólicos de las glándulas prostáticas en el proceso de carcinogénesis $^{29}$. Desgraciadamente aún así su positividad no puede considerarse enteramente específica del adenocarcinoma, dado que aunque de manera más débil, tiñe también casi todos los casos de neoplasia intraepitelial de alto grado, la atrofia parcial, algunos focos de adenosis, los focos de microacinos agrupados, la metaplasia nefrogénica e incluso algunas glándulas benignas ${ }^{30}$.

Únicamente un fenotipo con un marcador de células basales negativo y positivo para p504s debe sugerir malignidad ${ }^{31}$. Es por eso que recientemente se emplean con éxito combinaciones de doble inmunotinción de AMCR con p63 y/o 34bE12 $2^{32}$. Esta técnica de acoplamiento de dos anticuerpos al mismo tiempo reduce el número de cortes necesarios, el tiempo de técnica y en caso de ambigüedad el porcentaje de interpretaciones no concluyentes y de biopsias adicionales.

\section{BIBLIOGRAFÍA}

1. Rodríguez-Montes CE, Alvarado-Cabrero I. Nephrogenic adenoma of the urinary bladder. A lesion that can be confused with carcinoma. Report of 4 cases. Gac Med Mex 2001; 137: 67-72.

2. Peeker R, Aldenborg F, Fall M. Nephrogenoc adenoma: a study with special reference to clinical presentation. Br J Urol 1997; 80: 539542.

3. Gilcrease MZ, Delgado R, Vuitch F, AlboresSAAVEDRA J. Clear cell Adenocarcinoma and nephrogenic adenoma of the urethra and urinary bladder: a Histopathology and immunohistochemical comparison. Hum Pathol 1998; 29: 1451-1456.

4. Sousa Escandón MA, Pérez Valcárcel J, González Fernández M, González Rodríguez A, LAPEÑa Villarroya JA, Uribarri González C. Nephrogenic adenoma of the bladder: metaplasia or embryologic alteration? Actas Urol Esp 2001; 25: 307-311.

5. Friedman N, Kuhlenbeck H: Adenomatoid tumors of the urinary bladder reproducing renal structures (nephrogenic adenomas). J Urol 1950; 64: 657-670.

6. Bhagavan BS, Tiamson EM, Wenk RE, Berger BW, Hамамото G, Eggleston JC: Nephrogenic adenoma of the urinary bladder and urethra. J Hum Pathol 1981; 12: 907-916.

7. Sorensen FB, Jacobsen F, Nielsen JB, Mommsen S. Nephroid metaplasia of the urinary tract. A survey of the literature, with the contribution of 5 new immunohistochemically studies cases, including one case examined by electron microscopy. Acta Pathol Microbiol Immunol Scand A 1987; 95: 67-81.

8. Sikri K, Foster C, Machugh N, Marshall R. Localization of Tamm-Horsfall glicoprotein in the human kidney using immuno-fluorescence and immuno-electron microscopical techniques. J Anat 1981; 132: 597-605.

9. Pamplona M, Paniagua P, Gimeno F, Avila E, Extramiana J, González P, et al.: Adenoma nefrogénico en divertículo uretral femenino. Actas Urol Esp 1990; 14: 277-278.

10. Fournier G, Menut P, Moal MC, Hardy E, Volant A, Mangin P: Nephrogenic adenoma of the bladder in renal transplant recipients: a report of 9 cases with assessment of recipients of deoxyribonucleid acid ploidy and long term follow-up. J Urol 1996; 156: 41-44.

11. Husain AN, Armin AR, Schuster GA. Nephrogenic metaplasia of urinary tract in children: report of three cases and review of the literature. Pediatr Pathol 1988; 8: 293-300.

12. Oliva E. Young RH. Nephrogenic Adenoma of the urinary tract: A review of the microscopic appearance of 80 cases with emphasis on unusual features. Mod Pathol 1995; 8: 722-730.

13. Young RH, EblE JN. Non-neoplastic disorders of the urinary bladder. En:Bostwick DG $\mathrm{y}$ Eble JN Eds., Urologic Surgical Pathology, Mosby, St Louis, 1997.

14. Whang M, Katz L, Ongcapin E, Geffner S, Friedman G, Mulgaonkar $S$ et al. Nephrogenic adenomas occurring in a patient with simultaneous kidney-pancreas transplant. Urology 2000; 55: 949.

15. Ladenheim H, Frable W. Cytology of a recurrent nephrogenic adenoma in a renal transplant patient. Diagn Cytopathol 2009; 37: 468-470.

16. Kilcier M, Tan O, Ozgok Y, Tahmaz L, Deveci S, ERduran D. Nephrogenic adenoma of the bladder after intravesical bacillus Calmette Guerin treatment. Urol Int 2000; 64: 229-232.

17. Isimbaldi G, Di Nuovo F, Sironi M, Catania G, Bonacina R, Privitera O et al. Nephrogenic adenoma of the bladder. Morphological and 
immunophenotypic study with particular attention to differential diagnosis. Pathologica 1999; 91: 192-197.

18. Gilcrease MZ, Delgado R, Vuitch F, AlboresSAAVEDRA J. Clear cell adenocarcinoma and nephrogenic adenoma of the urethra and urinary bladder: A histopathologic and immunohistochemical comparison. Hum Pathol 1998; 29: 1451-1456.

19. MOLINÉ V, BAUMerT H. New markers in prostate biopsies. Actas Urol Esp 2007; 31: 1009-1024.

20. Molinie V. Minute focus, suspected focus andhigh-grade prostatic intraepithelial neoplasia. What is the significance? What are the consequences? Prog Urol 2004; 14: 2-6.

21. EPSTEIN JI. Diagnosis and reporting of limited adenocarcinoma of the prostate on neddle biopsy. Mod Pathol 2004; 17: 307-315.

22. Evans AJ. Alpha-methylacyl Coa racemasa (P504S): over-view and potential uses in diagnostic pathology as applied to prostate needle biopsies. J Clin Pathol 2003; 56: 892-897.

23. HAMmeEd O, Humphrey PA. Immunohistochemistry in diagnostic surgical pathology of the prostate. Semin Diagn Pathol 2005; 22: 88-104.

24. Carella R, Deleonardi G, D'Errico A, Salerno A, EgARTER-VIgL E, SEeBacher C et al. Immunohistochemical panels for differentiating epithelial malignant mesothelioma from lung adenocarcinoma: a study with logistic regression analysis. Am J Surg Pathol 2001; 25: 43-50.

25. Gown AM, Vogel AM. Monoclonal antibodies to human intermediate filament proteins. III. Analysis of tumors. Am J Clin Pathol 1985; 84: 413-424.
26. Yang XJ, Lecksell K, Gaudin P, Epstein JI. Rare expression of high-molecular-weight cytoqueratin in adenocarcinoma of the prostate gland: a study of 100 cases metastasic and locally advanced prostate cancer. Am J Surg Pathol 1999; 23: 147-152.

27. Varma M. JaSAnI B. Diagnostic utility of immunohistochemistry in morphologically difficult prostate cancer: review of current literature. Histopathology 2005; 47: 1-16.

28. Rubin MA, Zhou M, DhanaseKaran SM, Varambally S, Barrette TR, Sanda MG et al. AlphaMethylacyl coenzyme A racemasa as a tissue biomarker for prostate cancer. Jama 2002; 287: 1662-1670.

29. Davis LD, Zhang W, Merseburger A, Young D, Xu L, RHIM JS et al. p63 expression profile in normal and malignant prostate epithelial cells. Anticancer Res 2002; 22: 3819-3825.

30. Herawi M, Parwani AV, Irie J, Epstein JI. Small glandular proliferations on needle biopsies: most common benign mimickers of prostatic adenocarcinoma sent in for expert second opinion. Am J Surg Pathol 2005; 29: 874-880.

31. Zhou M, AydinH, Kanane H, Epstein JI. How often does alpha-methylacyl-CoA-racemasa contribute to resolving an atypical diagnosis on prostate neddle biopsy beyond that provided by basal cell markers? Am J Surg Pathol 2004; 28: 239-243.

32. Jiang Z, Li C, Fischer A, Dresser K, Woda BA. Using an AMACR (p504s)/34betaE12/p63 cocktail for the detection of small focal prostate carcinoma in neddle biopsy specimens. Am J Clin Pathol 2005; 123: 231-236. 